\title{
The prognostic values of m6A RNA methylation regulators in uveal melanoma
}

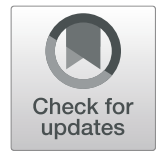

Jing Tang ${ }^{1+}$, Qi Wan ${ }^{1,2^{*}+}$ and Jianqun $\mathrm{Lu}^{1+}$

\begin{abstract}
Background: The aim of this study was to identify gene signatures and prognostic values of m6A methylation regulators in uveal melanoma (UM).

Methods: The RNA sequencing dataset and corresponding clinical information were downloaded from TCGA and GEO database. Based on the expression of m6A RNA methylation regulators, the patients were further clustered into different groups by applying the "ClassDiscovery" algorithm. Best survival analysis was performed to select prognostic m6A regulators and multivariate cox regression analysis was applied to constructed m6A regulators signature. The association between mutations and m6A regulators was assessed by Kruskal-Wallis tests and clinical characteristics were examined by using chi-square test.
\end{abstract}

Results: Totally, we identified two molecular subtypes of UM (C1/2) by applying consensus clustering to m6A RNA methylation regulators. In contrast to the C1 subtype, the C2 subtype associates with a better prognosis, has higher percentage of subtype 1 and lower percentage of Monosomy 3 which have been regarded as the well established prognostic markers for UM. The malignant hallmarks of mTORC1 signaling, oxidative phosphorylation, interferon-a response and apoptosis signaling are also significantly enriched in the C1 subtype. Moreover, a 3-m6A regulators signature was constructed by multivariate cox regression analysis method, which closely correlated with chromosome 3 status, subtype 1 of UM and can robustly predict patients' overall survival time.

Conclusions: m6A RNA methylation regulators take a crucial role in the potential malignant progression and prognostic value of UM and might be regarded as a new promising biomarker for UM prognosis and treatment strategy development.

Keywords: Uveal melanoma, m6A regulators, biomarker, Survival analysis

\section{Background}

Uveal melanoma is the most common type of malignant tumor of the adult eye, with an overall mortality rate of $50 \%[1,2]$. The prognosis for patients of UM remains poor, though advances in diagnosis and treatment have been reported [3, 4]. Therefore, it is important to

\footnotetext{
* Correspondence: 824314985@qq.com

${ }^{\dagger}$ Jing Tang, Qi Wan and Jianqun Lu these authors contributed equally as cofirst authors.

'Department of Ophthalmology, The People's Hospital of Leshan, city, Leshan, China

${ }^{2}$ State Key Laboratory of Ophthalmology, Zhongshan Ophthalmic Center,

Sun Yat-Sen University, Guangzhou 510064, China
}

explore the molecular mechanism underlying the survival events of UM and identify new prognostic factors and therapeutic targets.

It is well known that both DNA and histone proteins control gene expression by dynamic and reversible chemical modifications. RNA modification, like DNA and protein modification, is dynamically regulated by methyl-transferases [5]. The most prevalent RNA methylation is N6-methyladenosine (m6A), which exists in about $25 \%$ of transcripts at the genome-wide level and was firstly discovered in the 1970s. m6A RNA methylation regulators modify translocation, stability, RNA splicing and translation [6]. m6A is dynamically regulated

(c) The Author(s). 2020 Open Access This article is licensed under a Creative Commons Attribution 4.0 International License, which permits use, sharing, adaptation, distribution and reproduction in any medium or format, as long as you give appropriate credit to the original author(s) and the source, provide a link to the Creative Commons licence, and indicate if changes were made. The images or other third party material in this article are included in the article's Creative Commons licence, unless indicated otherwise in a credit line to the material. If material is not included in the article's Creative Commons licence and your intended use is not permitted by statutory regulation or exceeds the permitted use, you will need to obtain permission directly from the copyright holder. To view a copy of this licence, visit http://creativecommons.org/licenses/by/4.0/ The Creative Commons Public Domain Dedication waiver (http://creativecommons.org/publicdomain/zero/1.0/) applies to the data made available in this article, unless otherwise stated in a credit line to the data. 
by the 'writers' (RNA methyltransferases), such as METTL14, METTL3 and WTAP, is removed by 'erasers' (the demethylases), such as ALKBH5 and FTO, and 'readers' (the binding proteins), such as YTHDF2 and YTHDF1 [7]. RNA methyltransferases, the demethylases, and the binding proteins are often upregulated in a variety of human cancer types, increasing the expression of Oncogenes and Oncoproteins, augmenting the proliferation, progression, and metastasis of cancer cells [8].

m6A modification not only plays a vital role in the pathogenesis of a variety of human disease including obesity, neuronal disorders and immunological disease, but also has been shown to contribute to tumor initiation and promote progression of cancer and recurrence [9]. In addition, growing evidence suggests that gene mutation and abnormal expression of m6A regulators are intimately associated with the malignant progression of various cancers [10]. Although it is recognized that RNA methylation plays a critical role in different types of cancers, little is known about the relationship between m6A-related genes and UM.

Hence, in this study, we systematically evaluated the expression of m6A regulators in $80 \mathrm{UM}$ samples from The Cancer Genome Atlas (TCGA) dataset as well as the association between the genetic alterations and clinical characteristics and validation in $28 \mathrm{UM}$ samples from Gene Expression Omnibus (GEO) dataset. We found that the expression of $\mathrm{m} 6 \mathrm{~A}$ regulators plays critical roles in the malignant process of $\mathrm{UM}$, and we identified three $\mathrm{m} 6 \mathrm{~A}$ regulators as potential biomarkers through their prognostic signatures.

\section{Methods}

\section{Data processing}

The RNA sequencing and mutation expression dataset as well as the corresponding clinical information of 80 uveal melanoma patients were downloaded from TCGA (http://cancergenome.nih.gov/). GSE84976 dataset consist of 28 uveal melanoma patients obtained from GEO (https://www.ncbi.nlm.nih.gov/geo), which was used for validation dataset. Firstly, the probe IDs were be transformed into official gene symbols based on the platform. When multiple probe IDs were matched to the same gene symbol, the probe ID with max expression value was selected to represent that gene. Then, the raw matrix data were normalized by $\log 2(\mathrm{x}+1)$ conversion.

\section{Consensus clustering of $\mathrm{m} 6 \mathrm{~A}$ regulators}

We first selected thirteen m6A RNA methylation regulators from previously published articles $[7,9,10]$, and then we restricted the TCGA and GEO downloaded datasets to those features. Based on the expression of m6A regulators, we clustered the uveal melanoma patients into different subgroups. Principal component analysis was performed to determine whether these m6A regulators could definitely divide the samples into different uveal melanoma subgroups. To evaluate the interactive relationships among $\mathrm{m} 6 \mathrm{~A}$ regulators, correlation analyses of m6A regulators was applied and we also mapped the m6A regulators to the STRING database (http://stringdb.org). To investigate the pathways enriched in the different subgroups. we performed biology process (BP) and cancer hallmark pathway enrichment analysis. Firstly, patients of UM were classified into different subgroups and differentially analyses were calculated. Then, an ordered list of all genes was generated by the their log2 fold change value. GSEA was performed to assesses the functions associated with different subtypes.

\section{Prognostic signature building and risk survival analysis}

The association between the m6A regulators and overall survival (OS) of melanoma patients was analyzed. Best survival analysis was performed to select the prognostic m6A regulators. Then, multivariate cox regression analysis method was used to construct prognostic signature with the selected m6A regulators. For the risk formula and the risk score is generated as follows: Risk score $=$ $\sum_{i=1}^{\mathrm{N}}\left(\operatorname{coef}_{i} \times \operatorname{expr}_{i}\right)$. Based on the risk model, the risk score of each sample was calculated. The patients were divided into high-risk group and low-risk group by using the median cutoff of risk score. The survival curves of Kaplan-Meier were drawn and the differences among groups were compared by log-rank tests. The area under the curve (AUC) of ROC curve was used to evaluate the 5 -year overall survival predictive accuracy of the model.

\section{Statistical analysis}

All statistical analyses were conducted using R (v.3.5.2). The samples in UM were clustered by applying the "ClassDiscovery" package. GSEA analysis was performed by using "clusterProfiler" package. The statistical significance of risk score distribution including chromosome 3 status, subtype, SF3B1 status, BAP1 status and immune infiltration was estimated by Kruskal-Wallis tests. Differences in the expression of $\mathrm{m} 6 \mathrm{~A}$ regulators between mutant and wildtype of top 5 mutated genes were performed by multiple testing and the corrected $p$-value was calculated with the Benjamini-Hockberg method. The correlation coefficient of expression of m6A regulators was calculated by Spearman method. The associated between $\mathrm{m} 6 \mathrm{~A}$ regulatory genes and clinicopathological characteristics were analyzed with Fisher exact test or Pearson Chi-square test appropriately. Univariate and multivariate Cox regression analyses were performed to determine the prognostic value of the risk score and various clinical characteristics. The Kaplan-Meier survival analysis was applied to compare the overall survival 


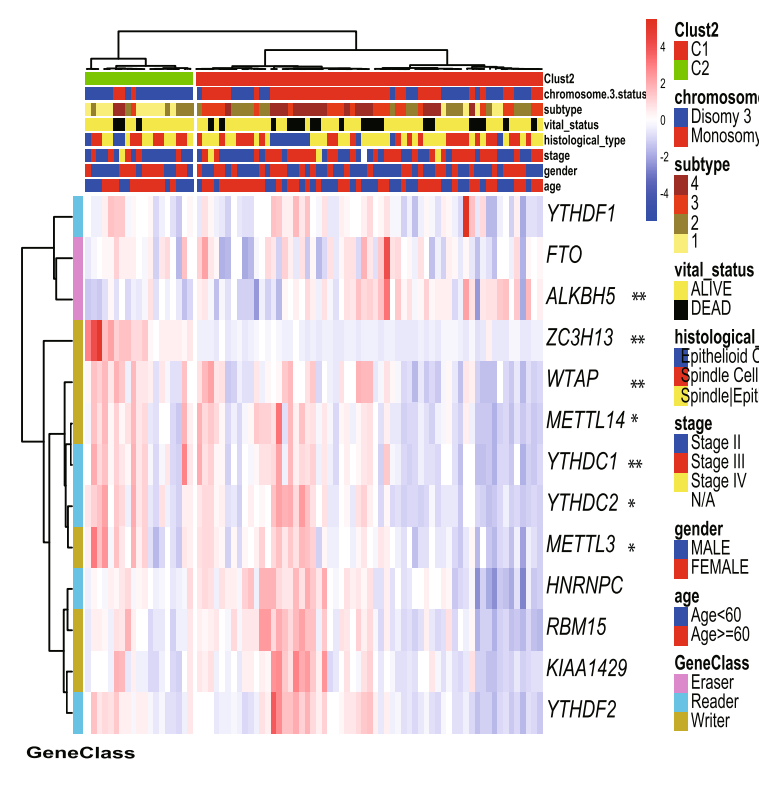

A

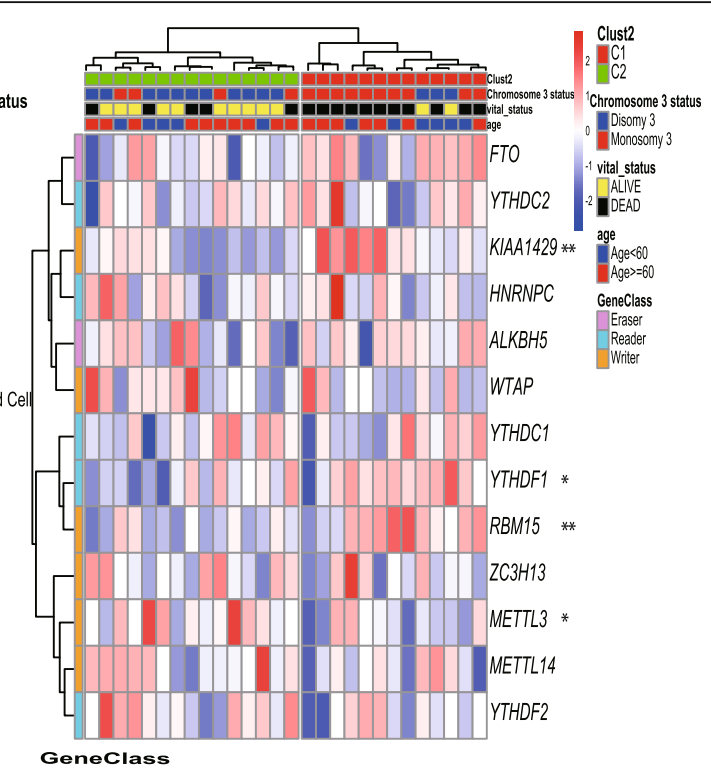

B
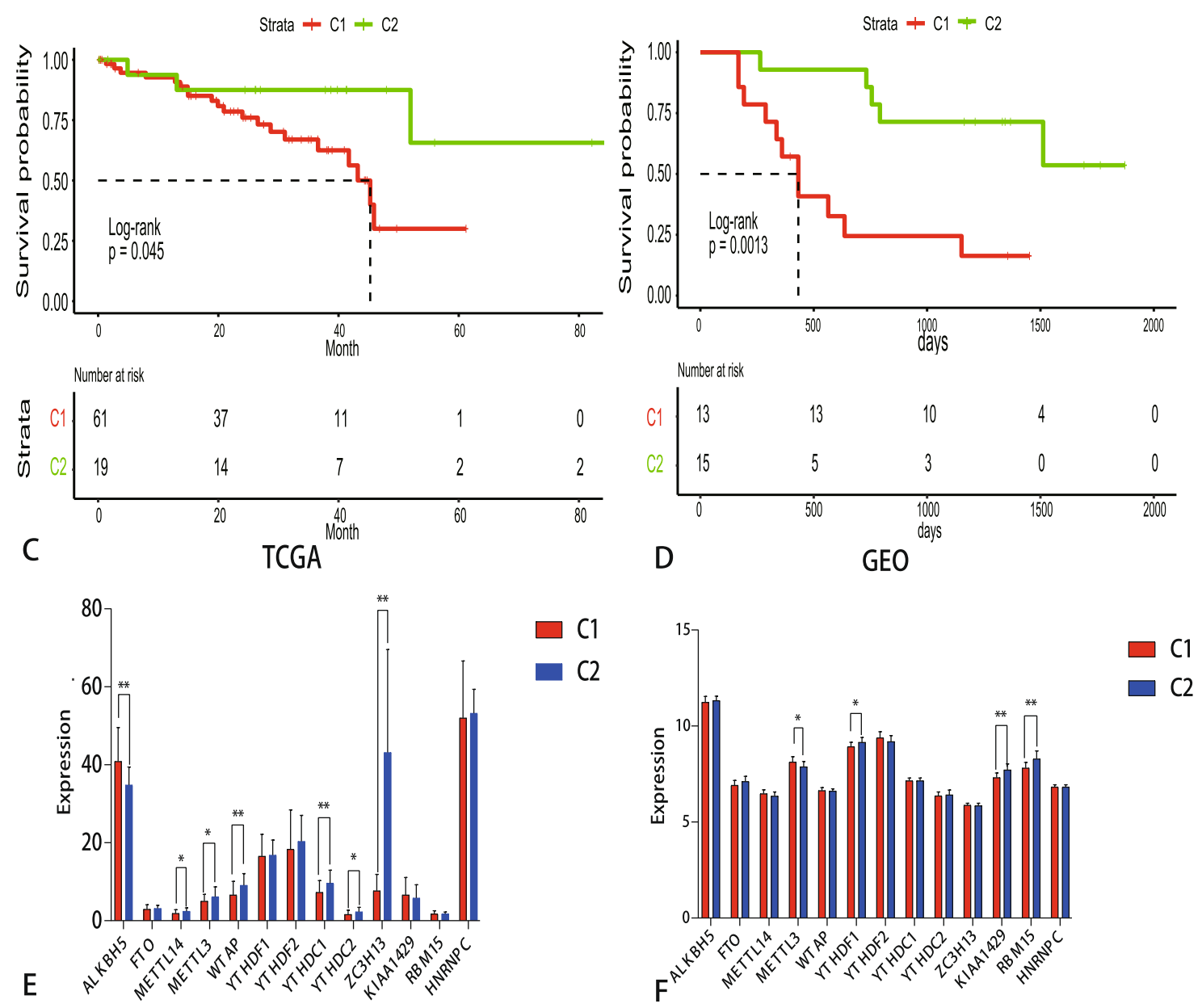

Fig. 1 (See legend on next page.) 
(See figure on previous page.)

Fig. 1 Expression of m6A RNA methylation regulators in uveal melanoma from the different database. a-b Heatmap and clinicopathologic features of the two clusters (C1/2) defined by the m6A RNA methylation regulators consensus expression downloaded from TCGA and GEO database. c-d Differential overall survival of uveal melanoma in the C1/2 subtypes (e-f) The mean expression levels of m6A RNA methylation regulators across the sample belonging to the $\mathrm{C} 1 / \mathrm{C} 2$ group. ${ }^{*} P<0.05,{ }^{* *} P<0.01$

Table 1 Clinicopathological characteristics of C1/2 molecular subtypes

\begin{tabular}{|c|c|c|c|c|c|}
\hline TCGA & level & C1 & $\mathrm{C} 2$ & $p$ & test \\
\hline$n$ & & 61 & 19 & & \\
\hline \multirow[t]{2}{*}{ age (\%) } & Age $<60$ & $27(44.3)$ & $9(47.4)$ & 1.000 & Chisq Test \\
\hline & Age $>=60$ & $34(55.7)$ & $10(52.6)$ & & \\
\hline \multirow[t]{2}{*}{ gender (\%) } & FEMALE & $25(41.0)$ & $10(52.6)$ & 0.529 & Chisq Test \\
\hline & MALE & $36(59.0)$ & $9(47.4)$ & & \\
\hline \multirow[t]{3}{*}{ M (\%) } & mo & $39(66.1)$ & $12(63.2)$ & 0.972 & Chisq Test \\
\hline & m1 & $3(5.1)$ & $1(5.3)$ & & \\
\hline & $\mathrm{mx}$ & $17(28.8)$ & $6(31.6)$ & & \\
\hline \multirow[t]{2}{*}{ N (\%) } & no & $40(65.6)$ & $12(66.7)$ & 1.000 & Chisq Test \\
\hline & $\mathrm{nx}$ & $21(34.4)$ & $6(33.3)$ & & \\
\hline \multirow[t]{3}{*}{ T (\%) } & t2 & $9(14.8)$ & $5(26.3)$ & 0.225 & Chisq Test \\
\hline & t3 & $23(37.7)$ & $9(47.4)$ & & \\
\hline & t4 & $29(47.5)$ & $5(26.3)$ & & \\
\hline \multirow[t]{4}{*}{ stage (\%) } & N/A & $1(1.6)$ & $0(0.0)$ & 0.238 & Chisq Test \\
\hline & Stage ॥ & $26(42.6)$ & $13(68.4)$ & & \\
\hline & Stage III & $31(50.8)$ & $5(26.3)$ & & \\
\hline & Stage IV & $3(4.9)$ & $1(5.3)$ & & \\
\hline \multirow[t]{3}{*}{ histological_type (\%) } & Epithelioid Cell & $10(16.4)$ & $3(15.8)$ & 0.888 & Chisq Test \\
\hline & Spindle Cell & $22(36.1)$ & $8(42.1)$ & & \\
\hline & Spindle Cell|Epithelioid Cell & $29(47.5)$ & $8(42.1)$ & & \\
\hline \multirow[t]{2}{*}{ vital_status (\%) } & ALIVE & $41(67.2)$ & $16(84.2)$ & 0.255 & Chisq Test \\
\hline & DEAD & $20(32.8)$ & $3(15.8)$ & & \\
\hline \multirow[t]{4}{*}{ subtype (\%) } & subtype1 & $5(8.2)$ & $10(52.6)$ & 0.000 & Chisq Test \\
\hline & subtype2 & $17(27.9)$ & $6(31.6)$ & & \\
\hline & subtype3 & $21(34.4)$ & $1(5.3)$ & & \\
\hline & subtype4 & $18(29.5)$ & $2(10.5)$ & & \\
\hline \multirow[t]{2}{*}{ chromosome.3.status (\%) } & Disomy 3 & $22(36.1)$ & $16(84.2)$ & 0.001 & Chisq Test \\
\hline & Monosomy 3 & $39(63.9)$ & $3(15.8)$ & & \\
\hline GEO & level & C1 & C2 & $p$ & test \\
\hline$n$ & & 15 & 13 & & \\
\hline \multirow[t]{2}{*}{ Age (\%) } & Age $<60$ & $8(53.3)$ & $4(30.8)$ & 0.412 & Fisher exact test \\
\hline & Age $>=60$ & $7(46.7)$ & $9(69.2)$ & & \\
\hline \multirow[t]{2}{*}{ vital_status (\%) } & ALIVE & $10(66.7)$ & $2(15.4)$ & 0.019 & Fisher exact test \\
\hline & DEAD & $5(33.3)$ & $11(84.6)$ & & \\
\hline \multirow[t]{2}{*}{ Chromosome.3.status (\%) } & Disomy 3 & $11(73.3)$ & $3(23.1)$ & 0.023 & Fisher exact test \\
\hline & Monosomy 3 & $4(26.7)$ & $10(76.9)$ & & \\
\hline \multirow[t]{2}{*}{ Metastasis (\%) } & No & $11(73.3)$ & $4(30.8)$ & 0.061 & Fisher exact test \\
\hline & Yes & $4(26.7)$ & $9(69.2)$ & & \\
\hline
\end{tabular}




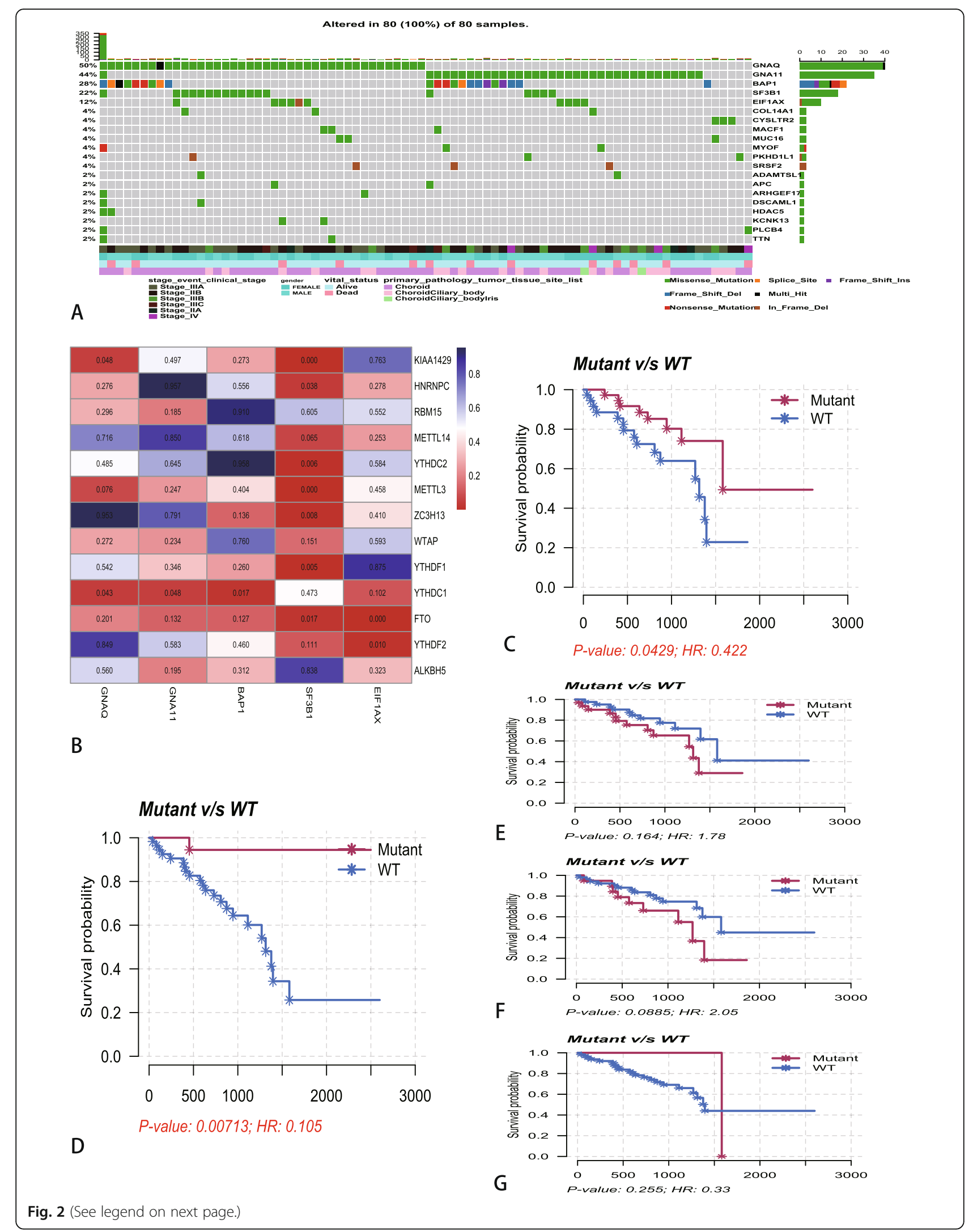


(See figure on previous page.)

Fig. 2 Relationships between mutated genes and mRNA expression levels of thirteen m6A regulators. a The waterfall plots of top 20 mutated genes in 80 UM samples at TCGA database. $\mathbf{b}$ The subgroup analysis the subgroup analysis of m6A regulators between mutant and wildtype of top 5 mutated genes. The blue and the red colors in heatmap represent higher and lower corrected $p$-value, respectively. c-g Kaplan-Meier survival analysis for GNAQ (c), SF3B1(d), GNA11(e), BAP1 (f) and EIF1AX (g) mutated genes

of the patients in the different groups or in the low- and high-risk groups. The hazard ratios (HR) and 95\% confidence intervals $(95 \% \mathrm{CI})$ of the prognostic factors were calculated. $P<0.05$ was regarded as statistically significant in all statistical tests.

\section{Results}

\section{Subgroup analysis of $\mathrm{m} 6 \mathrm{~A}$ regulators}

As a result, the expression of thirteen m6A RNA methylation regulators and clinicopathological characteristics associated to UM patients were obtained from TCGA and GEO. Based on "ClassDiscovery" algorithm, $80 \mathrm{UM}$ patients from TCGA and 28 UM patients from GEO can be identified two clusters of groups, respectively (Fig. 1a and $b$ ). Then, we contrasted the clinical features of these two subgroups, namely, $\mathrm{C} 1$ and $\mathrm{C} 2$. The subgroups analysis of clinical characteristics showed that Chromosome 3 status and subtype of UM have significant differences (Table 1). The others clinical characteristics like age, gender, TNM and stage have no statistical significance. To find out the potential correlation of overall survival with $\mathrm{C} 1$ and $\mathrm{C} 2$. Kaplan-Meier survival analysis was performed and the curves showed that overall survival of samples in $\mathrm{C} 2$ is longer than the samples in the $\mathrm{C} 1$ group (Fig. 1c, d). Then, expression levels of thirteen m6A RNA methylation regulators in UM patients with different $\mathrm{C} 1 / 2$ groups were shown in Fig. 1e, $\mathrm{f}$.

\section{Gene mutation and $\mathrm{m} 6 \mathrm{~A}$ regulators}

Then, we assessed the relationship between gene mutation and $\mathrm{m} 6 \mathrm{~A}$ regulators. we firstly identified the top 5 mutated genes based on the number of samples in which the genes are mutated in TCGA database, which was calculated by 'maftools' R package (Fig. 2a). The heatmap of differences in the expression of m6A regulators between mutant and wildtype of top 5 mutated genes indicated that SF3B1 was the most significantly regulated the expression of m6A regulators (Fig. 2b). The heatmap showed that the expression levels of ALKBH5, FTO, WTAP, YTHDF1, YTHDF2, YTHDC2 and KIAA1429 are significant differences between mutant-SF3B1 and wildtype-SF3B1. Kaplan-Meier analysis of these 5 mutant genes showed that only GNAQ (Fig. 2c) and SF3B1 (Fig. 2d) have significant differences with overall survival. As for GNA11 (Fig. 2e), BAP1 (Fig. 2f) and EIF1AX (Fig. 2g), the Kaplan-Meier analysis showed that there is on significantly differences between mutant and wildtype.

\section{Clustered molecular subtype of uveal melanoma}

The above results revealed that the clustered molecular subtype was intimately related to the prognosis of uveal melanoma. For better understanding of the interrelations among the thirteen m6A regulators, we also analyzed the interrelation (Fig. 3a) and correlation (Fig. 3c) among these regulators. ALKBH5 seems to be the hub gene of the 'Eraser', and correlated or co-expressed with METT L3, WTAP, YTHDF2, M ETTL14, YTHDF1, YTHDC1, YTHDC2, RBM15, KIAA1429. The correlation analysis showed that these regulators were significantly positively correlated with each other. Principal components analysis showed that $\mathrm{C} 1$ samples and C2 samples in TCGA datasets could be well differentiated based on the expression of m6A regulators (Fig. 3b). To investigate biologic pathways shared by the different $\mathrm{C} 1 / 2$ subtype, we performed GSEA analysis. According to the following criteria: $p$ value $<0.05$ and normalized enrichment score: | NES | $\geq 1.49$ BP terms were differentially enriched in $\mathrm{C} 1$ expression phenotype. The top $5 \mathrm{BP}$ terms indicated that pathways are commonly enriched $\mathrm{T}$ cell mediated pathways, including positive regulation of $\mathrm{T}$ cell mediated cytotoxicity, antigen processing and presentation of endogenous antigen, regulation of $\mathrm{T}$ cell mediated cytotoxicity, positive regulation of $\mathrm{T}$ cell mediated immunity and regulation of $\mathrm{T}$ cell mediated immunity (Fig. 3d). Moreover, the GSEA analysis of cancer malignant hallmarks of tumors showed that 9 terms including mTORC1 signaling, oxidative phosphorylation, interferon-a response and apoptosis signaling were significantly associated with the $\mathrm{C} 1$ subgroup expression phenotype (Fig. 3e).

\section{Identification and confirmation of $\mathrm{m} 6 \mathrm{~A}$ regulators signature}

For better predict the clinical and pathologic outcomes of UM with m6A regulators. Firstly, best survival analysis was used to evaluate associations between m6A regulators and OS in TCGA dataset. Totally, three m6A regulators were seeded out (Fig. 4a). Then, we used the three m6A regulators to constructed risk system by multivariate cox regression analysis. The risk system reckons a risk score for each patient. The distributions of the risk scores, OS, vital status, and expression levels of corresponding $3 \mathrm{~m} 6 \mathrm{~A}$ regulators in TCGA dataset were shown in Fig. 4b-d. Next, UM samples were divided into a high-risk group $(n=40)$ and a low-risk group $(n=40)$ by applying the median value of the risk scores. Kaplan-Meier curves revealed that low risk group 


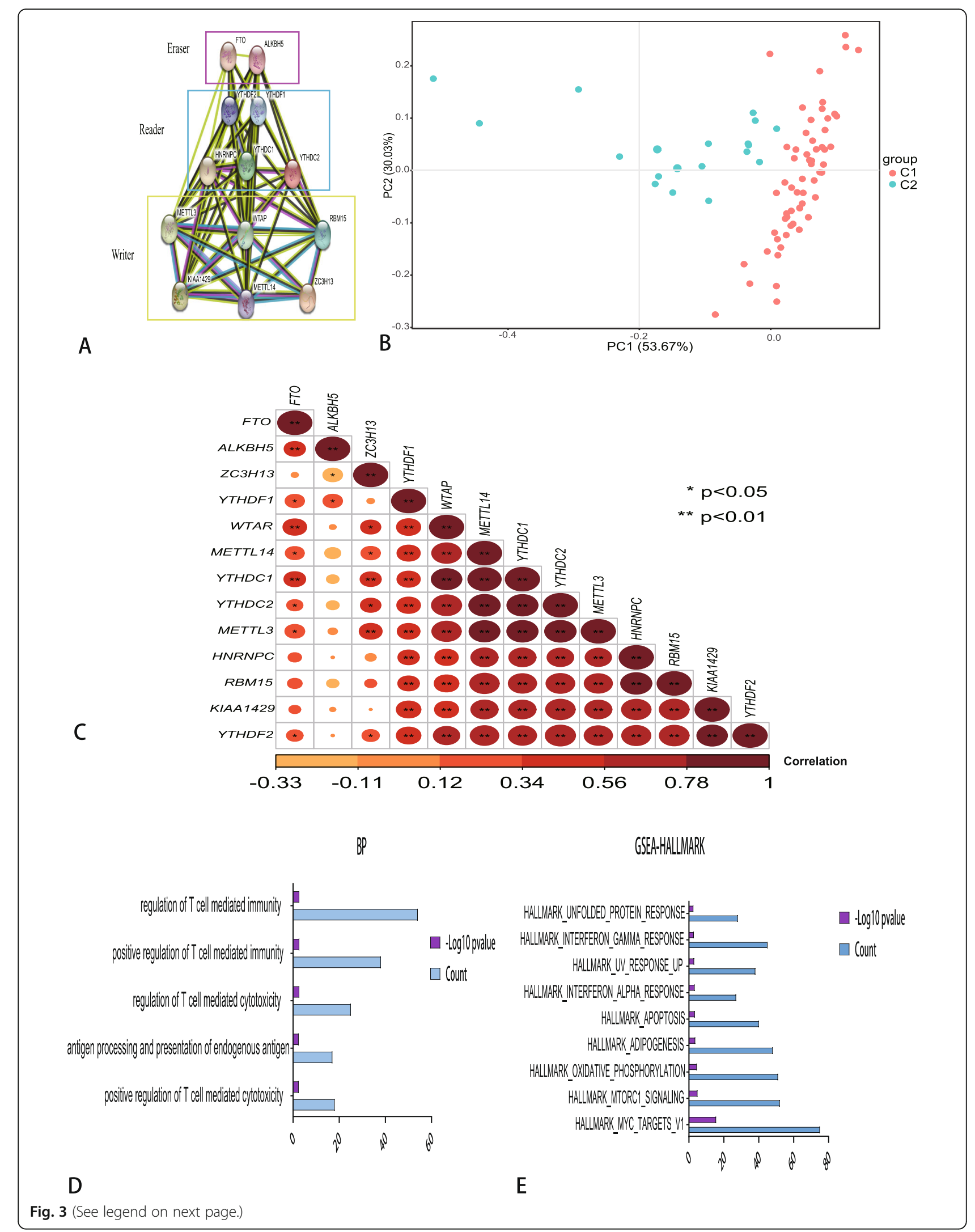


(See figure on previous page.)

Fig. 3 Interaction among m6A RNA methylation regulators and functional annotation of uveal melanoma in C1/2 subtypes. a The m6A modification-related interactions among the $13 \mathrm{~m} 6 \mathrm{~A}$ RNA methylation regulators. $\mathbf{b}$ Principal component analysis of the total RNA expression profile in the TCGA dataset. c Spearman correlation analysis of the $13 \mathrm{~m} 6 \mathrm{~A}$ modification regulators. ${ }^{*} P<0.05$, ${ }^{* *} P<0.01$. d The top 5 biology process (BP) terms were actively enriched in C1 expression phenotype. X-axis means the number of genes enriched in pathways, $\mathrm{Y}$-axis means $\mathrm{BP}$ pathways. $\mathbf{f}$ Cancer hallmark pathways revealed that 9 malignant hallmark pathways were significantly associated with the C1 subgroup expression phenotype. X-axis means the number of genes enriched in pathways, Y-axis means hallmark pathways

have a significant longer survival time than high risk (Fig. 4e). The ROC curve showed that the 5 years of AUC was 0.645 (Fig. 4f). To verify the predictive ability of the three $\mathrm{m} 6 \mathrm{~A}$ regulators, validation analysis was performed in GEO dataset. The distributions of the risk scores, OS, vital status, and expression levels of corresponding $3 \mathrm{~m} 6 \mathrm{~A}$ regulators in GEO dataset were shown in Fig. 4g-i. The curve of Kaplan-Meier revealed that there is a significant difference between high-risk and low-risk group with log-rank test of $p=0.044$ (Fig. 4j). The 5 years of AUC was 0.677 (Fig. 4k). The subgroups analysis of clinical characteristics between low- and high- risk groups showed that chromosome 3 status, subtype, and vital status in TCGA and GEO have significant differences (Table 2).

\section{Associations between risk score and clinical variables}

The associations between risk score of $\mathrm{m} 6 \mathrm{~A}$ regulators and clinical variables such as chromosome 3 status, mutated SF3B1, mutated BAP1 and subtype were explored. The box plots showed that monosomy 3 have higher risk scores than disomy 3 (Fig. 5a), wildtype of SF3B1 own higher risk scores than mutant (Fig. 5b), and subtype 4 of UM have the highest scores than other subtypes (Fig. 5d). While, subgroup of mutated BAP1 manifested that there is on significant difference between mutant and wildtype (Fig. 5c). To evaluate the associations between risk score and immune microenvironment, "MCPcounter" package in $\mathrm{R}$ was applied to calculate the immune scores of immune cells. Subgroup analysis of immune cells showed that significant differences were founded in T cells, CD8 T cells, cytotoxic lymphocytes, natural killer (NK) cells, monocytic lineage and myeloid dendritic cells between high and low risk subgroups (Fig. 5e). Besides, univariate and multivariate logistic regression were used to compare the prognostic value of risk score and other clinical variables in TCGA and GEO datasets. The forest plots indicated that age, stage, histology, subtype, chromosome 3 status, metastasis and risk sore were significantly associated with OS in univariate analysis, but only the risk score were significantly correlated with OS in multivariate analysis (Fig. 5f-g). The 5 years AUC of age, stage, histology, subtype, chromosome 3 status and risk score in TCGA were $0.591,0.535,0.351,0.788,0.791$ and 0.645 respectively
(Fig. 5h). As for GEO, the 5 years AUC of chromosome 3 status and risk score were 0.698 and 0.677 (Fig. 5i).

\section{Discussion}

The growing genome-wide studies demonstrated that most of the human genome is transcribed, which exists a complex network consist of large and small RNA molecules in human cells. However, only 1 to $2 \%$ of the transcripts have the capacity for protein translation [1113]. In fact, post-transcriptional regulation at the RNA level through cis-and trans-mechanisms is essential to control the gene expression procedures that determine cellular function and cell fate [14]. To date, more than 150 chemical modifications have been described for RNA. Among them, m6A is the most prevalent posttranscriptional modification of eukaryotic mRNAs and long noncoding RNAs. Recent studies have indicated that $\mathrm{m} 6 \mathrm{~A}$ regulators have been shown to play important regulatory roles in diverse biological processes in human cancer [15]. However, despite of the increasing evidence for their implication in cancers, the potential role of m6A regulators in UM prognosis is little known about.

In this study, we demonstrated that the expression of m6A regulators is also intimately related to the prognosis and malignancy of UM. Based on the expression of m6A regulators, we identified two UM subgroups, namely $\mathrm{C} 1 / 2$ molecular subgroup, by applying consensus clustered method. The C1/2 molecular subgroup not only affected the clinical and prognosis features but also closely associated with biological signals and malignant hallmarks of UM. Survival analysis showed that C1 subgroup have worse overall survival than $\mathrm{C} 2$ subgroup. In addition, $\mathrm{C} 1$ subgroup have higher percentage of subtype 4 which have been proven the worst outcome subgroup in previous UM TCGA study [16]. GSEA analysis showed that positive regulation of $\mathrm{T}$ cell mediated pathways and malignant hallmarks such as mTORC1 signaling, oxidative phosphorylation, interferon-a response and apoptosis signaling positively activate in $\mathrm{C} 1$ subgroup. In fact, T cells like active CD $4+$ and $\mathrm{CD} 8+$ cells have antitumor immunity and therapy functions [17]. As to $\mathrm{C} 1$ molecular subtype, lots of malignant hallmark of pathways were enriched. Thus, it is reasonable to believe that clustered molecular subtypes $\mathrm{C} 1 / 2$ are closely correlated to the malignancy and prognosis of UM. Moreover, extensive researches also suggest that UM with 


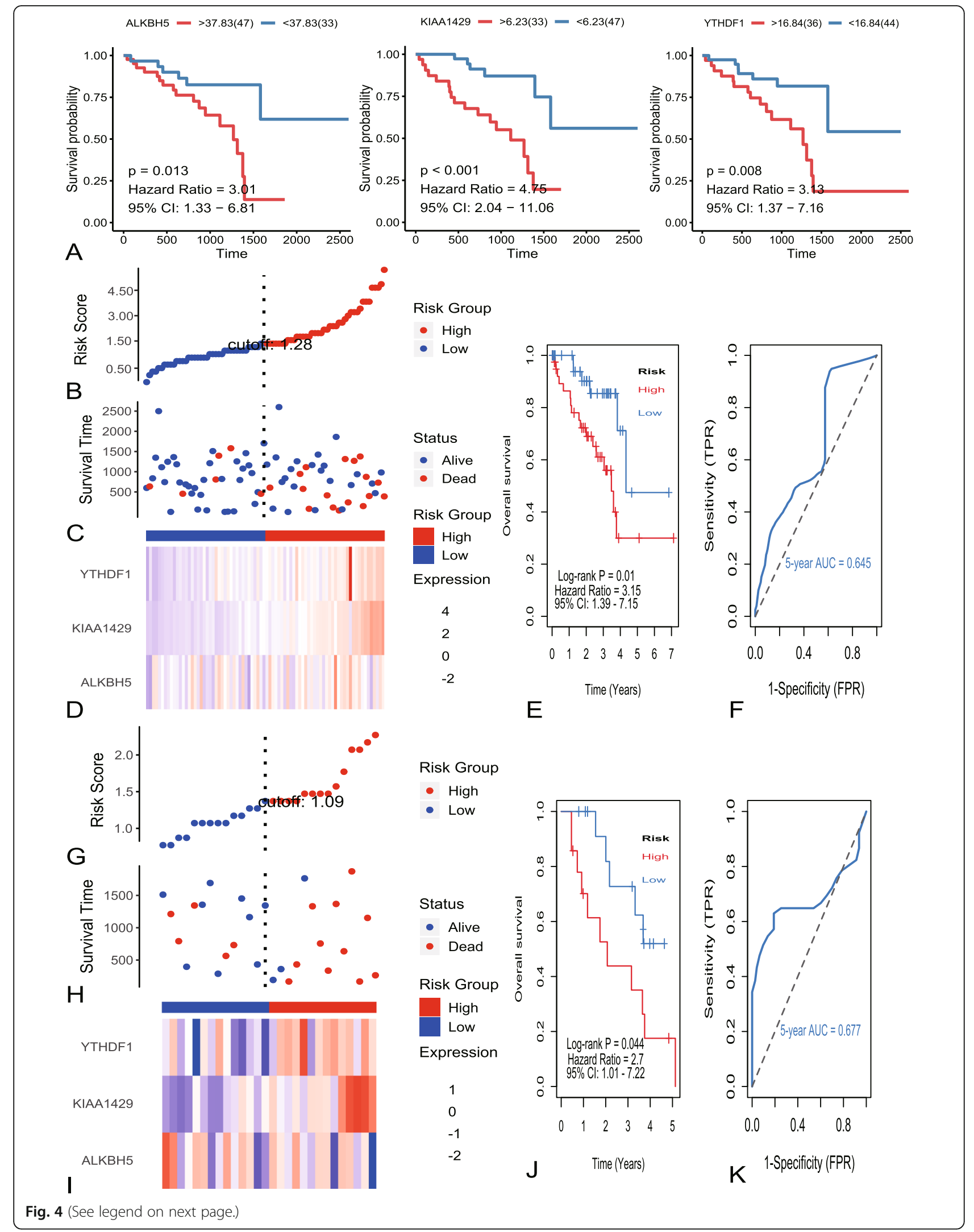


(See figure on previous page.)

Fig. 4 Identification and validation of m6A regulators signature. a Kaplan-Meier survival curves of ALKBH5, YTHDF1 and KIAA1429. b The distribution of risk score in TCGA dataset. The risk scores are arranged in ascending order from left to right. c The overall survival (OS) and vital status of patients. $\mathbf{d}$ The expression patterns of three identified m6A regulators for 80 patients in TCGA. e Kaplan-Meier survival curves of patients in the high-risk and low-risk groups. $\mathbf{f}$ The 5 years of the receiver operating characteristic (ROC) curve in TCGA dataset. $\mathbf{g}$ The distribution of risk score in GEO dataset. The risk scores are arranged in ascending order from left to right. $\mathbf{h}$ The overall survival (OS) and vital status of patients. $\mathbf{i}$ The expression patterns of three identified $\mathrm{m} 6 \mathrm{~A}$ regulators for 28 patients in GEO. $\mathbf{j}$ Kaplan-Meier survival curves of patients in the high-risk and low-risk groups. $\mathbf{k}$ The 5 years of the receiver operating characteristic (ROC) curve in GEO dataset

Table 2 The subgroups analysis of clinical characteristics between low- and high-risk groups

\begin{tabular}{|c|c|c|c|c|c|}
\hline TCGA & level & High_risk & Low_risk & $p$ & test \\
\hline$n$ & & 40 & 40 & & \\
\hline \multirow[t]{2}{*}{ age (\%) } & Age $<60$ & $16(40.0)$ & $20(50.0)$ & 0.500 & Chisq Test \\
\hline & Age $>=60$ & $24(60.0)$ & $20(50.0)$ & & \\
\hline \multirow[t]{2}{*}{ gender (\%) } & FEMALE & $14(35.0)$ & $21(52.5)$ & 0.176 & Chisq Test \\
\hline & MALE & $26(65.0)$ & $19(47.5)$ & & \\
\hline \multirow[t]{3}{*}{ M (\%) } & mo & $25(64.1)$ & $26(66.7)$ & 0.588 & Chisq Test \\
\hline & m1 & $3(7.7)$ & $1(2.6)$ & & \\
\hline & $\mathrm{mx}$ & $11(28.2)$ & $12(30.8)$ & & \\
\hline \multirow[t]{2}{*}{ N (\%) } & no & $25(64.1)$ & $27(67.5)$ & 0.935 & Chisq Test \\
\hline & $\mathrm{nx}$ & $14(35.9)$ & $13(32.5)$ & & \\
\hline \multirow[t]{3}{*}{ T (\%) } & t2 & $5(12.5)$ & $9(22.5)$ & 0.440 & Chisq Test \\
\hline & t3 & $18(45.0)$ & $14(35.0)$ & & \\
\hline & t4 & $17(42.5)$ & $17(42.5)$ & & \\
\hline \multirow[t]{4}{*}{ stage (\%) } & $\mathrm{N} / \mathrm{A}$ & $1(2.5)$ & $0(0.0)$ & 0.481 & Chisq Test \\
\hline & Stage II & $20(50.0)$ & $19(47.5)$ & & \\
\hline & Stage III & $16(40.0)$ & $20(50.0)$ & & \\
\hline & Stage IV & $3(7.5)$ & $1(2.5)$ & & \\
\hline \multirow[t]{3}{*}{ histological_type (\%) } & Epithelioid Cell & $10(25.0)$ & $3(7.5)$ & 0.010 & Chisq Test \\
\hline & Spindle Cell & $9(22.5)$ & $21(52.5)$ & & \\
\hline & Spindle Cell|Epithelioid Cell & $21(52.5)$ & $16(40.0)$ & & \\
\hline \multirow[t]{2}{*}{ vital_status (\%) } & ALIVE & $23(57.5)$ & $34(85.0)$ & 0.014 & Chisq Test \\
\hline & DEAD & $17(42.5)$ & $6(15.0)$ & & \\
\hline \multirow[t]{4}{*}{ subtype (\%) } & subtype1 & $3(7.5)$ & $12(30.0)$ & 0.000 & Chisq Test \\
\hline & subtype2 & $9(22.5)$ & $14(35.0)$ & & \\
\hline & subtype3 & $10(25.0)$ & $12(30.0)$ & & \\
\hline & subtype4 & $18(45.0)$ & $2(5.0)$ & & \\
\hline \multirow[t]{2}{*}{ chromosome.3.status (\%) } & Disomy 3 & $12(30.0)$ & $26(65.0)$ & 0.004 & Chisq Test \\
\hline & Monosomy 3 & $28(70.0)$ & $14(35.0)$ & & \\
\hline GEO & level & High_risk & Low_risk & $\mathrm{p}$ & test \\
\hline$n$ & & 14 & 14 & & \\
\hline \multirow[t]{2}{*}{ Age (\%) } & Age $<60$ & $5(35.7)$ & $7(50.0)$ & 0.703 & Fisher exact test \\
\hline & Age $>=60$ & $9(64.3)$ & $7(50.0)$ & & \\
\hline \multirow[t]{2}{*}{ vital_status (\%) } & ALIVE & $3(21.4)$ & $9(64.3)$ & 0.052 & Fisher exact test \\
\hline & DEAD & $11(78.6)$ & $5(35.7)$ & & \\
\hline \multirow[t]{2}{*}{ Chromosome.3.status (\%) } & Disomy 3 & $4(28.6)$ & $10(71.4)$ & 0.053 & Fisher exact test \\
\hline & Monosomy 3 & $10(71.4)$ & $4(28.6)$ & & \\
\hline \multirow[t]{2}{*}{ Metastasis (\%) } & No & $5(35.7)$ & $10(71.4)$ & 0.130 & Fisher exact test \\
\hline & Yes & $9(64.3)$ & $4(28.6)$ & & \\
\hline
\end{tabular}



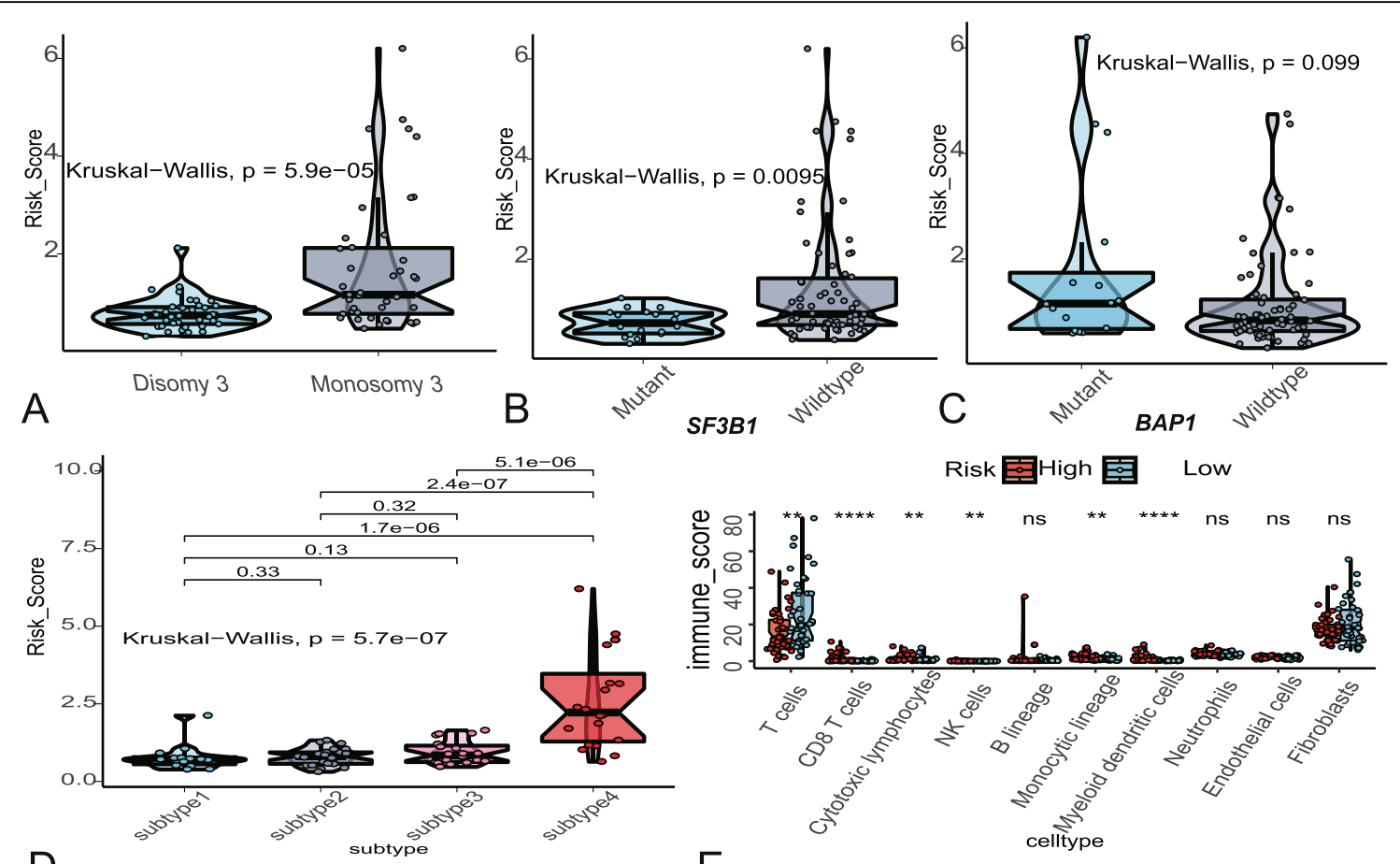

D

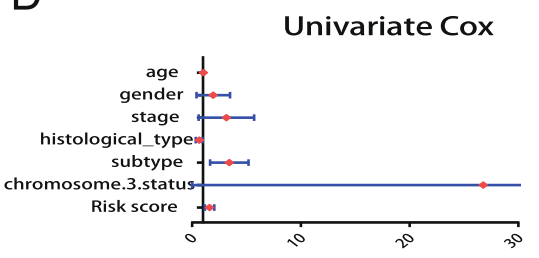

F

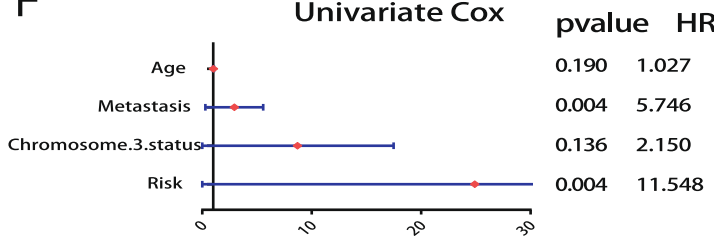

G

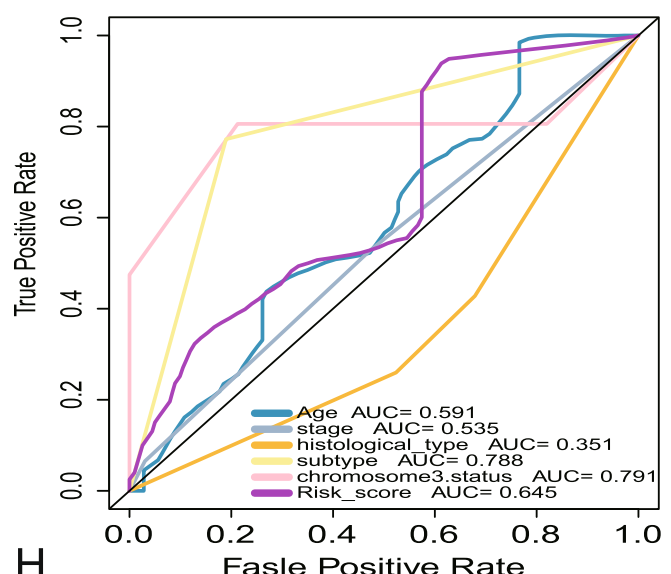

$\mathrm{H}$

Fasle Positive Rate

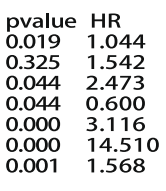

SF3B1

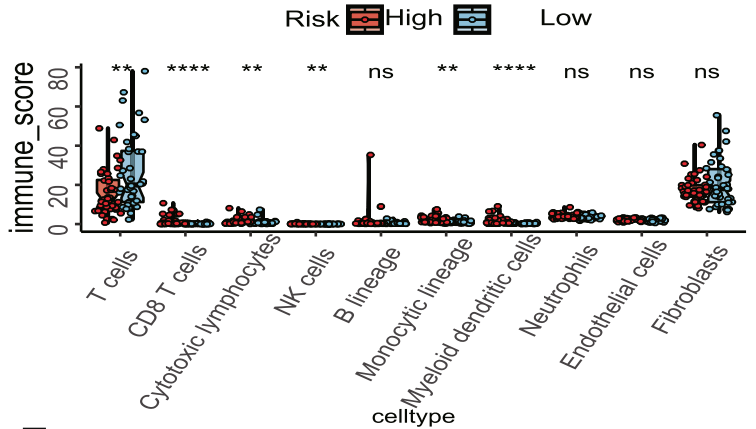

E

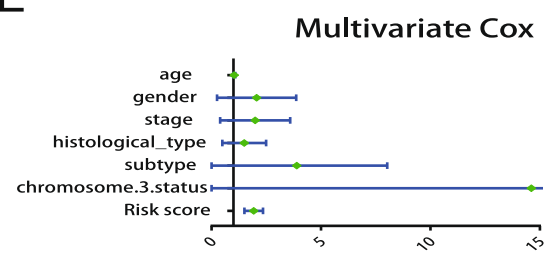

pvalue HR $0.058 \quad 1.040$ $0.394 \quad 1.531$ $0.316 \quad 1.566$ $\begin{array}{ll}0.507 & 1.272\end{array}$

$\begin{array}{ll}0.164 & 2.440 \\ 0.286 & 3.667\end{array}$ $\begin{array}{ll}0.286 & 3.667 \\ 0.025 & 1.804\end{array}$

Multivariate Cox
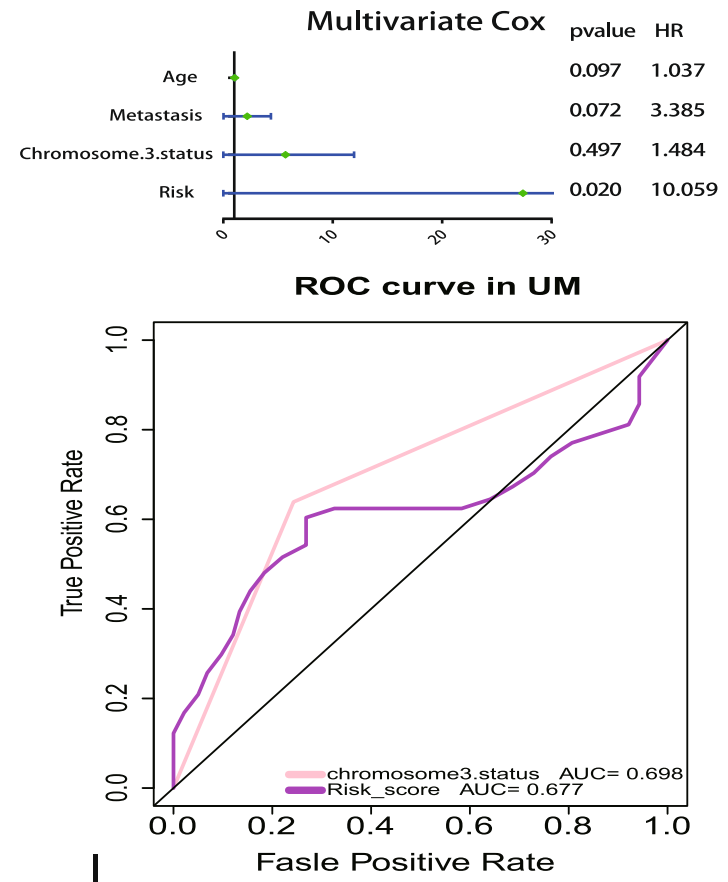

Fig. 5 (See legend on next page.) 
(See figure on previous page.)

Fig. 5 Associations between risk score and clinical variables. a-d The relationship between risk score distribution and clinical variables which contained chromosome 3 status (a), SF3B1 mutated types (b), BAP1 mutated types (c) and subtype (d). e The different immune scores of 10 immune cells between the high and low risk UM patients. $\mathbf{f}$ Forest plots of risk score and clinical variables in TCGA dataset. $\mathbf{g}$ Forest plots of risk score and clinical variables in GEO dataset. $\mathbf{h}$ The 5 years area under the curve (AUC) of risk score and clinical variables associated with OS in TCGA. $\mathbf{i}$ The 5 years AUC of risk score and clinical variables associated with OS in GEO

monosomy 3 is associated with a dramatically poor prognosis, which consistent with our research [18-20]. The subgroups analysis of Chromosome.3.status showed that the percentage of Monosomy 3 in C1 molecular subgroup is much higher than this in $\mathrm{C} 2$ molecular subtype (Table 1 ). The different analysis of m6A regulators between $\mathrm{C} 1 / 2$ molecular subtype showed that "eraser" like ALKBH5, "writer" like METTL3, METTL14, and WTAP and "readers", like YTHDF1 and YTHDF2 have significant differences. (Fig. 1e, f). The different expression of these m6A regulators may eventually lead to the various of survival outcomes [21]. Among the m6A regulators, previous studies indicated that the eraser ALKBH5 can induce breast cancer stem cell and glioblastoma stem-like cell proliferation and tumor initiation, [22] the writers METTL3 and METTL14 were reported to enhance glioblastoma growth and suppress Liver cancer metastasis, [23-25] the reader YTHDF1 and YTHDF2 induce cancer cell proliferation in colon cancer and lung oncogenic effects [26, 27]. These findings manifested that high or low expression of specific m6A regulators are related to misregulation of RNAs in tumors, and the same $\mathrm{m} 6 \mathrm{~A}$ regulator may take different functions in various tumors [28, 29].

By analyzing the mutation annotation files of the TCGA-UVM cohort, we identified 5 highly variant mutated genes and SF3B1 is the most significantly influence the expression of $\mathrm{m} 6 \mathrm{~A}$ regulators. SF3B1 (splicing factor 3subunit B1) mutations can be generally found in 10 to $21 \%$ of cases of UM. Previous researches have shown that SF3B1 mutations in UM patients are associated with favorable prognosis [30]. Survival analysis also indicated that SF3B1-mutated UM had a better survival than the SF3B1 wild-type. In our research, the results showed that the mutation of SF3B1 will generally significantly down-regulated the expression of m6A regulators, including "eraser" such as ALKBH5 and FTO; "writer" such as WTAP and KIAA1429; "reader" such as YTHD F1, YTHDF2 and YTHDC2 (Fig. 2b). Therefore, it easily envisaged that the mutant of SF3B1 may lead to downregulate the expression of "eraser" such as ALKBH5 and FTO and finally result in a better survival in UM.

What's more, we also distinguished a prognostic risk signature with three identified m6A regulators (ALKBH5, YTHDF1 and KIAA1429), which divided the overall survival of UM into high- and low-risk subgroups. KaplanMeier analyses indicated that high-risk subgroups with a poor survival. Stratified analysis of clinical characteristics between low- and high- risk groups also revealed that lots of risk factors like mortality rate, subtype 4 and monosomy 3 are take higher percentage in high-risk group (Table 2). Furthermore, UM patients in high risk group had higher immune infiltration than low risk group. The risk sores of monosomy 3, SF3B1-wildtype, and subtype 4 were respectively higher than disomy 3, SF3B1-mutated and subtype 1 in UM, which was consistent with previous researches. Notably, compared with the 5 years AUC values of previous prognostic markers (stage, subtype and chromosome 3 status), our signature can achieve similar accuracy value. Besides, only the risk score had significant associations with OS no matter in univariate or multivariate regression analysis. In sum, the signature we constructed might be regarded as a new promising biomarker which supply more simple and accurate clinical applications. For example, in human breast cancer cells, knockdown ALKBH5 contributed to significantly decrease the number of cancer stem cells and the opportunity of tumorigenesis. In addition, the high expression of ALKBH5 in glioblastoma can lead to stem-like cell proliferation and tumorigenesis [31].

\section{Conclusions}

In summary, we firstly comprehensively evaluated the expression, potential function, and prognostic value of m6A regulatory genes in UM from TCGA dataset and have validated in GEO dataset, which should be helpful for UM early diagnosis and might be regarded as a new promising biomarker for UM prognosis and treatment.

\begin{abstract}
Abbreviations
TCGA: The Cancer Genome Atlas database; GEO: Gene Expression Omniniub; UM: Uveal melanoma; GO: Gene ontology; BP: Biology process;

DEGs: Differently expressed genes; OS: Overall survival; AUC: The area under the curve; ROC: Receiver operating characteristic curves
\end{abstract}

\section{Acknowledgments}

Not applicable.

\section{Authors' contributions}

JT designed the study. QW and JQL wrote the paper. All authors read and approved the final manuscript.

\section{Funding}

There is no sponsorship or funding arrangements relating to our research.

\section{Availability of data and materials}

The datasets used and analysed during the current study available from the corresponding author on reasonable request. 


\section{Ethics approval and consent to participate}

No permissions were required to use the repository data.

\section{Consent for publication}

Not applicable.

\section{Competing interests}

All authors declare that they have no competing interests.

Received: 29 July 2019 Accepted: 9 July 2020

Published online: 18 July 2020

\section{References}

1. Stang A, Parkin DM, Ferlay J, Jockel KH. International uveal melanoma incidence trends in view of a decreasing proportion of morphological verification. Int J Cancer. 2005;114(1):114-23.

2. Singh AD, Turell ME, Topham AK. Uveal melanoma: trends in incidence, treatment, and survival. Ophthalmology. 2011;118(9):1881-5.

3. Rietschel P, Panageas KS, Hanlon C, Patel A, Abramson DH, Chapman PB. Variates of survival in metastatic uveal melanoma. J Clin Oncol. 2005;23(31): 8076-80.

4. Bol KF, Mensink HW, Aarntzen EH, Schreibelt G, Keunen JE, Coulie PG, de Klein A, Punt CJ, Paridaens D, Figdor CG, et al. Long overall survival after dendritic cell vaccination in metastatic uveal melanoma patients. Am J Ophthalmol. 2014;158(5):939-47.

5. Yu J, Chen M, Huang H, Zhu J, Song H, Zhu J, Park J, Ji S-J. Dynamic $m<$ sup $>6</$ sup $>$ A modification regulates local translation of mRNA in axons (Article). Nucleic Acids Res. 2018;46(3):1412-23.

6. Erson-Bensan AE, Begik O. m6A modification and implications for microRNAs. Microrna. 2017:6(2):97-101.

7. Vu LP, Cheng Y, Kharas MG. The Biology of m6A RNA Methylation in Normal and Malignant Hematopoiesis. Cancer Discovery. 2019;9(1):25-33.

8. Chai RC, Wu F, Wang QX, Zhang S, Zhang KN, Liu YQ, Zhao Z, Jiang T, Wang YZ, Kang CS. m(6) a RNA methylation regulators contribute to malignant progression and have clinical prognostic impact in gliomas. Aging. 2019;11(4):1204-25.

9. Lan Q, Liu PY, Haase J, Bell JL, Huttelmaier S, Liu T. The critical role of RNA m(6) a methylation in Cancer. Cancer Res. 2019;79(7):1285-92.

10. Sun T, Wu R, Ming L. The role of m6A RNA methylation in cancer. Biomedicine \& pharmacotherapy = Biomedecine \& pharmacotherapie. 2019; 112:108613.

11. Joshi P, Kooshki M, Aldrich W, Varghai D, Zborowski M, Singh AD, Triozzi PL. Expression of natural killer cell regulatory microRNA by uveal melanoma cancer stem cells. Clin Exp Metastasis. 2016;33(8):829-38.

12. Larsen AC, Holst L, Kaczkowski B, Andersen MT, Manfe V, Siersma VD, Kolko $\mathrm{M}$, Kiilgaard JF, Winther O, Prause JU, et al. MicroRNA expression analysis and multiplex ligation-dependent probe amplification in metastatic and non-metastatic uveal melanoma. Acta Ophthalmol. 2014:92(6):541-9.

13. Starkey MP, Compston-Garnett L, Malho P, Dunn K, Dubielzig R. Metastasisassociated microRNA expression in canine uveal melanoma. Vet Comp Oncol. 2018;16(1):81-9.

14. Kwok CT, Marshall AD, Rasko JE, Wong JJ. Genetic alterations of $\mathrm{m}(6)$ a regulators predict poorer survival in acute myeloid leukemia. J Hematol Oncol. 2017;10(1):39.

15. Yang Y, Hsu PJ, Chen YS, Yang YG. Dynamic transcriptomic m(6) a decoration: writers, erasers, readers and functions in RNA metabolism. Cell Res. 2018;28(6):616-24.

16. Robertson AG, Shih J, Yau C, Gibb EA, Oba J, Mungall KL, Hess JM, Uzunangelov V, Walter V, Danilova L, et al. Integrative analysis identifies four molecular and clinical subsets in Uveal melanoma. Cancer Cell. 2018;33(1):151.

17. Kummer M, Schuler-Thurner B. Immunotherapy of Uveal Melanoma: Vaccination Against Cancer. Methods Mol Biol. 2017;1499:273-8.

18. Aziz H, Salvi S, Singh N, Dar S, Hayden B, Singh A. Uveal Melanoma Regression After Brachytherapy: Relationship with Monosomy Chromosome 3. Investigative Ophthalmol Visual Sci. 2015;56(7):5346.

19. Hamadeh F, Medina CA, Singh AD, Reynods JP, Biscotti CV. Uveal melanoma: An analysis of cellular features and comparison to monosomy 3 status. Diagn Cytopathol. 2016;44(5):377-83.

20. Tura A, Merz H, Reinsberg M, Lüke M, Jager MJ, Grisanti S, Lüke J. Analysis of monosomy-3 in immunomagnetically-isolated circulating melanoma cells in uveal melanoma patients. Pigment Cell Melanoma Res. 2016;29(5):583-9.
21. Zhou J, Wang J, Hong B, Ma K, Xie H, Li L, Zhang K, Zhou B, Cai L, Gong K. Gene signatures and prognostic values of $\mathrm{m} 6 \mathrm{~A}$ regulators in clear cell renal cell carcinoma - a retrospective study using TCGA database. Aging. 2019; 11(6):1633-47.

22. Zhang S, Zhao BS, Zhou A, Lin K, Zheng S, Lu Z, Chen Y, Sulman EP, Xie K, Bogler O, et al. m(6) a Demethylase ALKBH5 maintains Tumorigenicity of Glioblastoma stem-like cells by sustaining FOXM1 expression and cell proliferation program. Cancer Cell. 2017;31(4):591-606 e596.

23. Barbieri I, Tzelepis K, Pandolfini L, Shi J, Millán-Zambrano G, Robson SC, Aspris D, Migliori V, Bannister AJ, Han N, : et al: Promoter-bound METTL3 maintains myeloid leukaemia by m6A-dependent translation control. Nature 2017:552;(7683):126-131.

24. Lin S, Choe J, Du P, Triboulet R, Gregory Rl. The $m<$ sup $>6</$ sup $>A$ Methyltransferase METTL3 Promotes Translation in Human Cancer Cells (Article). Molecular Cell. 2016:62(3):335-45.

25. Lin S, Choe J, Du P, Triboulet R, Gregory RI. The m6A Methyltransferase METTL3 Promotes Translation in Human Cancer Cells. Mol Cell. 2016;62(3): $335-45$.

26. Chen M, Wei L, Law $C T$, Tsang FHC, Shen J, Cheng CLH, Tsang LH, Ho DWH, Chiu DKC, Lee JMF, et al. RNA N6-methyladenosine methyltransferase-like 3 promotes liver cancer progression through YTHDF2-dependent posttranscriptional silencing of SOCS2. Hepatology. 2018;67(6):2254-70.

27. Tanabe A, Tanikawa K, Tsunetomi M, Takai K, Ikeda H, Konno J, Torigoe T, Maeda H, Kutomi G, Okita K, et al. RNA helicase YTHDC2 promotes cancer metastasis via the enhancement of the efficiency by which HIF-1a mRNA is translated (Article). Cancer Letters. 2016;376(1):34-42.

28. Li Z, Weng H, Su R, Weng X, Zuo Z, Li C, Huang H, Nachtergaele S, Dong L, $\mathrm{Hu}$ C, et al. FTO Plays an Oncogenic Role in Acute Myeloid Leukemia as a $\mathrm{N}<$ sup $>6</$ sup $>-$ Methyladenosine RNA Demethylase (Article). Cancer Cell. 2017;31(1):127-41.

29. Nishizawa Y, Konno M, Asai A, Koseki J, Kawamoto K, Miyoshi N, Takahashi H, Nishida N, Haraguchi N, Sakai D, et al. Oncogene c-Myc promotes epitranscriptome m6A reader YTHDF1 expression in colorectal cancer. Oncotarget. 2017;9(7):7476-86.

30. Yavuzyigitoglu S, Koopmans AE, Verdijk RM, Vaarwater J, Eussen B, van Bodegom A, Paridaens D, Kili E, de Klein A. Uveal Melanomas with SF3B1 Mutations. Ophthalmology. 2016;123(5):1118-28.

31. Zhang C, Zhi WI, Lu H, Samanta D, Chen I, Gabrielson E, Semenza GL. Hypoxia-inducible factors regulate pluripotency factor expression by ZNF217- and ALKBH5-mediated modulation of RNA methylation in breast cancer cells. Oncotarget. 2016;7(40):64527-42.

\section{Publisher's Note}

Springer Nature remains neutral with regard to jurisdictional claims in published maps and institutional affiliations.

Ready to submit your research? Choose BMC and benefit from:

- fast, convenient online submission

- thorough peer review by experienced researchers in your field

- rapid publication on acceptance

- support for research data, including large and complex data types

- gold Open Access which fosters wider collaboration and increased citations

- maximum visibility for your research: over $100 \mathrm{M}$ website views per year

At BMC, research is always in progress.

Learn more biomedcentral.com/submissions 